\title{
An Innovative Control Architecture for Autonomous Mobile Robots by Displacement of Computing Operations to Operator Stations
}

\author{
Muhammad Fahd Waseem
}

\begin{abstract}
This paper explores the practical implementation of a control architecture for autonomous mobile robots by avoiding the concentration of computing operations on the robot, and instead performing them at an operator station over a wireless link. By using this innovative approach, the cost of implementation may also be reduced.
\end{abstract}

Index Terms - Intelligent robots, wireless LAN, displaced computing, telerobotics.

\section{INTRODUCTION}

There is a wide variety of control architectures for mobile robots [1]. Most of them, however, tend to place a lot of computing power on the robot itself, particularly in the case of autonomous robots. The problem with this is that as the capabilities of the robot are expanded, the computing power needed to make intelligent decisions grows. Given the low volumetric space and weight restrictions imposed on most mobile robotics applications, for example, Urban Search and Rescue (USAR) or industrial robotics, the increased computing power must be packaged into smaller sizes. Not only is this an expensive approach, it is also relatively more difficult to troubleshoot issues and / or issue overrides (in case of fully autonomous robots).

An alternative approach is to use a robust wireless network and displace as much of the computing power as possible to an operator / base station and perform all the computationally intensive operations there. In this architecture, raw sensory data is transmitted from the robot, computation and decision making is performed remotely, and the control instructions are sent back to the robot. This architecture was prototyped and successfully implemented in "SAVIOUR", a robotics platform for competition in the USAR, Real Rescue Robot League of the RoboCup 2009, as well as the RoboCup IranOpen 2010[2], [3].

\section{BASICS OF THE ARCHITECTURE}

\section{A. Generic Case}

In the most generic case, completely raw data from sensors and nodes is taken and transmitted over a simple wireless link to a base station. The data is processed by a computer, and

Manuscript received August 4, 2012; revised September 20, 2012. This work was supported in part by the Ghulam Ishaq Khan Institute of Engineering Sciences and Technology (GIKI), Pakistan.

M. F. Waseem is with the the Faculty of Electronic Engineering, GIKI (e-mail: muhammadfahd@ieee.org). relevant control instructions are transmitted to the robot. These instructions may be decoded using simple circuitry for actuation purposes. See Fig.1.

\section{B. Optimization of Generic Case}

It may be inefficient to transmit completely raw data over a simple wireless link. Optimization of this architecture may be done by pre-processing and compressing the data to be transmitted [4]. Any multiplexing that needs to be done may also be performed. It may also be advantageous to choose a wireless link based on standard IEEE specifications e.g. IEEE 802.11g. See Fig. 2.

The advantages of such optimization include:

- Efficient utilization of wireless bandwidth

- More compact circuitry through use of microcontrollers

- Faster control response

- Lower power consumption

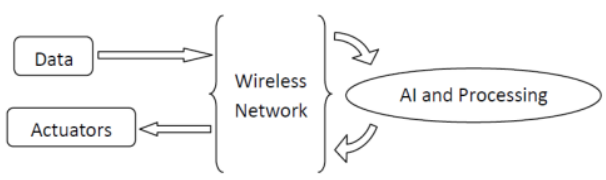

Fig. 1. Generic case.

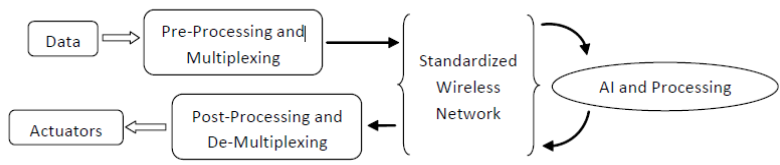

Fig. 2. Optimized generic case.

\section{ADVANTAGES OVER ON-BOARD COMPUTING METHODS}

The advantages of the given architecture over on-board computing methods are significant.

- Robots can be smaller due to the exclusion of onboard computers, because wireless transceiver equipment is usually less voluminous than embedded computers. Though computing devices have shrunk in size and weight in recent years, using wireless equipment to untether the computing power still leads to advantages [5].

- Regular personal computers (PCs) can be used to perform any audio-video or sensory data processing tasks that would otherwise have to be done on-board.

- Manual overrides can be added to the control software in case of malfunctioning artificial intelligence (AI). 
- Cost benefits also exist in replacing powerful on-board embedded computers with equally powerful desktop type computers at an operator station.

- The given architecture leads to reduced complexity on board the robot, which improves troubleshooting.

- If all processing was on-board, any issues with AI would require retrieval of robots from the field of operation. With the given architecture, that would not be necessary.

\section{IMPLEMENTATION}

\section{A. Data}

The data to be collected from the robot consisted of readouts from multiple sensors. These included thermal readings from medium range thermopile arrays, $\mathrm{CO} 2$ levels, audio and video.

\section{B. Pre-Processing}

Minor computational resources were used on-board to compress the data. For audio and video, a stock IP-camera was used. Simple logic circuitry was used to read and multiplex data from the sensors and a TCP/IP stack was used to convert the serial data into TCP packets [6].

\section{Wireless Link and Displaced Computing}

Because all data was in TCP packets, a wireless router placed on board the robot was used to set up the wireless link. A laptop computer with a wireless LAN card served as the computing base station. Both were $2.4 \mathrm{GHz}$ devices, adhering to the IEEE $802.11 \mathrm{~g}$ specifications. Software on the laptop served as the AI, with all video processing done in real time. Based on results from the full sensory processing, control instructions were issued via the wireless link.

The wireless router was an off-the-shelf device by the wireless equipment manufacturer, D-Link ${ }^{\mathrm{TM}}$. An omni-directional antenna was used with the wireless router, though a unidirectional antenna could also be used in most practical scenarios.

In actuality, the network access could be extended over the internet, allowing the AI operation to be displaced to practically anywhere in the world. As an application, it may be possible to deploy cheap, low volume robots over the world, and then reconfigure the AI on a central AI server to perform a myriad of different tasks.

\section{Actuation}

Data received over the wireless link was decoded into serial data using TCP/IP stacks. This data was interpreted as actuation commands by simple logic circuitry.

\section{E. AI Algorithm}

An AI algorithm was implemented at the base station using the $\mathrm{C \#}$ and MATLAB programming languages. This involved Simultaneous Localization and Mapping (SLAM) to autonomously chart a path towards a detected human survivor in a real, simulated earthquake hit arena. Obstacle avoidance and terrain mapping was also implemented, as was motion detection. The detection of human survivors was done primarily using thermal signatures. The AI would then direct the all-terrain, differential drive, tracked robot towards the victim for closer scrutiny and possible environmental manipulation.

Much of this was dependent on live video feed from cameras on the robot. If the video processing required for the successful implementation of the AI been done on-board the robot, it would have necessitated a larger, more expensive, robot or increased functional complexity.

A functional override was also provided. In the case that the AI repeatedly failed to perform the required tasks, a human operator could direct the robot manually.

\section{EXPERIMENTAL RESULTS}

Pertinent parameters were investigated to verify the functionality of the given control architecture. Of highest significance in such architecture are the video deterioration and lag factors caused by the introduction of the wireless link to the base station. It may be noted that the $9600 \mathrm{bps}$ serial channel used for the binary data from the sensors had little to no effect on the overall lag in comparison to the lag introduced by the video.

\section{A. Video Deterioration}

The compressed video feed was a single MPEG video stream of VGA resolution $(640 \times 480)$ and approximately 30 frames per second (fps). This frame rate began to drop at LOS ranges above 50 meters to an unusable $5 \mathrm{fps}$ at about 100 meters due to dropping network throughput rates. Air temperature was approximately $20^{\circ} \mathrm{C}$ during the testing.

\section{B. Lag Determination}

The lag between the appearance of an identifiable signature and the subsequent execution of control instructions by the robot was determined during testing by the simple method of using a screen in front of the thermal signature of the survivor. As a method, the screen was placed around the signature and then suddenly removed so that the signature would "appear" to the robot. In this way, a net measure of lag was defined as the time taken from the appearance of the signature to the start of movement of the robot towards the heat source.

This lag was measured at various distances. Two control sets were used:

1) Direct interfacing of the sensors and camera to the processing computer without a wireless link and the lag associated with the running of the algorithm. This method is an approximation to the traditional approach for autonomous robots: placing the computing power onboard the robot.

2) Using the architecture presented in this paper, but placing the robot only 1 meter away from the base station. This would simulate near ideal, short distance, LOS network throughput rates.

Lag times were then measured at actual competitive distances between the robot and the base station. All testing was done at direct LOS. Table I summarizes the results. 
TABLE I: EXPERIMENTAL PERFORMANCE RESULTS

\begin{tabular}{lcc}
\hline \hline Range Set & $\begin{array}{l}\text { Average Video } \\
\text { Framerate (fps) }\end{array}$ & $\begin{array}{l}\text { Average Lag Time } \\
\text { (milliseconds) }\end{array}$ \\
\hline $\begin{array}{l}\text { Control 1: Direct } \\
\text { Interfacing }\end{array}$ & 30 & 400 \\
Control 2: Zero Range & 30 & 401 \\
20 meters & 30 & 405 \\
30 meters & 29 & 407 \\
40 meters & 27 & 409 \\
50 meters & 25 & 420 \\
60 meters & 21 & 435 \\
80 meters & 13 & 446 \\
100 meters & 6 & 472 \\
\hline \hline
\end{tabular}

\section{INTERPRETATION OF RESULTS}

It may be noted from the results that:

- Video frame rates drop sharply after 50 metres of range.

- Lag does not increase very sharply as the range exceeds 50 meters. This is due to the fact that very little data is needed for signature detection and just a few frames of video are enough to start navigation. Repeated correction of route is performed as data flows in. Furthermore, the majority of the time is used for processing, not for transmission.

Usable range is mainly limited by the dropping video frame rates. The software algorithm used in our particular case ceases to function properly below $5 \mathrm{fps}$.

\section{LIMITATION AND DISADVANTAGES}

Most of the main limitations and disadvantages of the architecture stem from the reliance on a robust network connection between the robot and the base station.

As can be seen from the results, satisfactory operation of the autonomous robot is dependent on a certain range from the base of operation. This effectively limits the field of operations of the robot to a particular maximum radius originating at the base station. As a corollary, wireless connection strength must be sampled to prevent the robot from wandering too far off.

Fallback measures in case of network failure have to be implemented on-board the robot; though in practical cases, this should normally consist of nothing more than halting all motion of the robot and waiting for resumption in network connectivity.

Network interference may also cause issues, as with any wireless network [7].

All these limitations and disadvantages can be mitigated or reduced by following standard techniques for increasing the range and robustness of networks [8], [9], [10], [11].

\section{CONCLUSION}

Displacing computing operations to a base station over a wireless network link in case of fully autonomous robots offers the advantages of more compact robots, less bulk, possibility of manual override and lower costs. It is easy to replace complex circuitry on-board the robot with simple circuitry, and move complex computing operations to a larger, cheaper, powerful computer at the base station by using a standardized wireless network. The limitations of being dependent on a wireless network are offset by the benefits, and the limitations themselves are easy to increase using standard network improvement techniques.

\section{ACKNOWLEDGEMENTS}

The author would like to thank his team who helped him complete the other aspects of the "SAVIOUR" robot which was used for the testing of the architecture presented in this paper. The author would also like to thank Dr. Junaid Mughal, Dr. Shafaat Bazaz and Mr. Muhammad Farooq without whom this work would not have been possible.

\section{REFERENCES}

[1] A. A. D. Medeiros, "A survey of control architectures for autonomous mobile robots," Journal of the Brazilian Computer Society, vol. 4, no. 3, Apr 1998.

[2] Robocup 2009 | teams. Graz University of Technology. (2010, Jun) [Online]. Available: http://www.robocup2009.org/302-0-teams

[3] Robocup iranopen $2010>$ leagues $>$ robocup rescue $>$ rescue robot league > teams. Islamic Azad University of Qazvin. (2010, Jun) [Online]. Available: http://www.iranopen2010.ir/Default.aspx?tabid=132\&language=en-G B

[4] A. K. Jain, "Image data compression: A review," Proc. IEEE, vol. 69, no. 3, pp. 349 - 389, Mar 1981.

[5] R. H. Katz, "Adaptation and mobility in wireless information systems," IEEE Commun. Mag., vol. 40, no. 5, pp. 102-114, may 2002.

[6] A. Dunkels, "Full tcp/ip for 8-bit architectures," in MobiSys '03: Proceedings of the 1st International Conference on Mobile systems, applications and services. New York, USA: ACM, 2003, pp. 85-98.

[7] S. Kawade and T. Hodgkinson, "Analysis of interference effects between co-existent $802.11 \mathrm{~b}$ and $802.11 \mathrm{~g}$ wi-fi systems," in Vehicular Technology Conference, 2008. VTC Spring 2008. IEEE, vol. 11-14, pp. 1881-1885, 2008.

[8] C. Chiasserini and R. Rao, "Coexistence mechanisms for interference mitigation in the 2.4-ghz ism band," IEEE Trans. Wireless Commun., vol. 2, no. 5, pp. 964-975, Sep 2003.

[9] K. Kowalik, M. Bykowski, B. Keegan, and M. Davis, "Practical issues of power control in ieee 802.11 wireless devices," in Telecommunications, 2008. ICT 2008. International Conference on, Jun 2008, pp. 1-5.

[10] V. Mhatre, K. Papagiannaki, and F. Baccelli, "Interference mitigation through power control in high density 802.11 wlans," in INFOCOM 2007. 26th IEEE International Conference on Computer Communications. IEEE, May 2007, pp. 535-543.

[11] G. Zaggoulos and A. Nix, "Wlan/wds performance using directive antennas in highly mobile scenarios: Experimental results," in Wireless Communications and Mobile Computing Conference, 2008 IWCMC '08. International, Aug 2008, pp. 700-705.

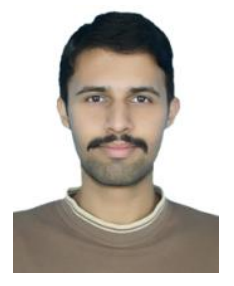

Muhammad F. Waseem was born on $10^{\text {th }}$ January, 1989. He comes from a family of medical doctors, but decided to overturn this trend by graduating with top honours from GIKI, Pakistan, with a Bachelors of Science (Electronics Engineering). He specializes in robotics and applicative engineering, i.e. the solution of industrial problems in-situ. He is currently employed at Schlumberger Limited, Pakistan, where he is working in the Testing Services Segment as Field Engineer. He maintains his personal websites at muhammadfahd.com and muhammadfahd.wordpress.com. 